\title{
Guías de experiencia de usuario para aplicaciones de turismo cultural basadas en realidad aumentada
}

\section{User experience guidelines to cultural tourism applications based on augmented reality}

\author{
Micaela Vega, Gabriela Gaetan, Adriana Martin \\ vegamicaela97@outlook.es,ggaetan@uaco.unpa.edu.ar,amartin@uaco.unpa.edu.ar \\ Universidad Nacional de la Patagonia Austral (UNPA) \\ Unidad Académica Caleta Olivia (UACO) - Instituto de Tecnología Aplicada (ITA)
}

Recibido: 05/05/2021. Aceptado: 25/10/2021

\begin{abstract}
RESUMEN
En los últimos años, la Realidad Aumentada se ha aplicado en diferentes ámbitos relacionados a patrimonio cultural, incluyendo ambientes interiores, como museos y galerías y ambientes al aire libre, como guías turísticos. La Realidad Aumentada proporciona una tecnología que aplicada al desarrollo de productos útiles y fáciles de usar, mejora la experiencia de usuario de los turistas. En este trabajo, proponemos un conjunto preliminar de guías de Experiencia de Usuario para aplicaciones móviles de Turismo Cultural, basadas en Realidad Aumentada. La propuesta se basa en: la revisión de estudios existentes sobre Experiencia de Usuario en productos de software para Turismo Cultural con Realidad Aumentada, y también en el análisis de expertos en Experiencia de Usuario. Como trabajo futuro, pretendemos validar el conjunto de guías obtenidas en casos de estudio.
\end{abstract}

Palabras clave: Patrimonio Cultural; Aplicaciones Móviles; Experiencia de Usuario; Realidad Aumentada.

\begin{abstract}
In recent years, Augmented Reality has been applied in different areas related to cultural heritage, including indoor environments, such as museums and galleries, and outdoor environments, such as tourist guides. Augmented Reality provides a technology that, applied to the development of useful and easy-to-use products, improves the user experience of tourists. In this work, we propose a preliminary set of User Experience guides for Cultural Tourism mobile applications, based on Augmented Reality. The proposal is based on: the review of existing studies on User Experience in software products for Cultural Tourism with Augmented Reality, and also on the analysis of experts in User Experience. As future work, we intend to validate the set of guides obtained in case studies.
\end{abstract}

Keywords: Cultural Heritage; Mobile Applications; User Experience; Augmented Reality.

\section{INTRODUCCIÓN}

Una de las definiciones más populares, dice que se considera Experiencia de Usuario (UX) a "Las percepciones y respuestas de una persona que resultan del uso y / o uso anticipado de un producto, sistema o servicio" (ISO/TC 159/SC 4, 2010). 
El diseño de UX, se basa en principios de diseño, heurísticas, pautas, mejores prácticas, patrones o estándares de diseño de interfaces de usuario (Masip, Martinie, Winckler, Palanque, Granollers, \& Oliva, 2012), que sirven a diseñadores y evaluadores de UX. Según (Kenneth, 1997), una guía sirve como base para el desarrollo y es una colección de principios, directrices y convenciones; los principios son los objetivos que guían las decisiones de diseño; las convenciones son decisiones de diseño específicas que se eligen para una organización particular; mientras que las directrices, se basan en los principios pero se especifican para reflejar las necesidades de un dominio particular.

En la literatura se pueden encontrar trabajos que abordan el diseño de UX tanto para aplicaciones de Patrimonio Cultural, como para aplicaciones de Realidad Aumentada. Estas recomendaciones varían en nivel de detalle, formato, alcance, lenguaje, y clasificación; lo que significa que los diseñadores deben combinar las diferentes fuentes de recomendaciones, resolviendo conflictos entre las guías identificadas.

Las recomendaciones más conocidas son las pautas de usabilidad universal de Nielsen (Nielsen, 1994); sin embargo éstas no cubren todos los aspectos involucrados. Se necesitan guías adicionales para respaldar componentes y funcionalidades específicas del Turismo Cultural, como la precisión del contenido, la posibilidad de ofrecer contenido en varios idiomas, y presentar contenido atractivo para el usuario, entre otras. Además, existen algunos factores que son especialmente importantes sobre la tecnología de RA.

Estas recomendaciones se aplican en etapas iniciales de diseño y desarrollo, para validar las decisiones de diseño y garantizar una buena experiencia de usuario.

El propósito de este trabajo es favorecer la comprensión de aspectos para diseñar experiencia de usuario, en un dominio de aplicación específico. Para esto, en base a trabajos anteriores (Vega, Gaetan, Martin, \& Saldaño, 2020), (Cardozo, Martin, Saldaño, \& Gaetán, 2020), desarrollamos un conjunto de guías de diseño UX para aplicaciones móviles de Patrimonio Cultural con Realidad Aumentada.

Este documento se ha estructurado de la siguiente manera: La Sección 2 se enfoca en la Realidad Aumentada. En la Sección 3, se detallan aspectos de Turismo Cultural. Por su parte, en la Sección 4 se describen los Materiales y Métodos de este estudio, y los Resultados en la Sección 5. Finalmente, en la Sección 6, se desarrollan las conclusiones.

\section{REALIDAD AUMENTADA}

La Realidad Aumentada (RA) tiene como objetivo mejorar la percepción e interacción del usuario con el entorno real agregando información virtual. La definición que propone Azuma (Azuma, 1997) dice que la RA es "la combinación de elementos reales y virtuales, la cual es además interactiva en tiempo real y está registrada en 3D". En un sistema de RA al mundo real se le agregan objetos virtuales como texto, imágenes $2 \mathrm{D}$, imágenes $3 \mathrm{D}$, o audios, permitiendo que el usuario interactúe con la realidad. Estos objetos virtuales se deben vincular espacialmente con el mundo real de forma coherente y precisa.

Por otra parte, la Realidad Virtual (RV), sumerge completamente al usuario en un mundo virtual sin ninguna posibilidad de ver el entorno real, excepto a través de representaciones generadas por computadora (Carmigniani, Furht, Anisetti, Ceravolo, Damiani, \& Ivkovic, 2011). 
Las diferencias entre RA y RV están dadas principalmente por el tipo de interacción con el entorno. En este sentido, Milgram y Kishino (Milgram \& Kishino, 1994) definen el Diagrama de Continuidad Realidad-Virtualidad, que se muestra en la Figura 1, como una escala continua que oscila entre lo que se define como lo completamente virtual hasta lo que es completamente del entorno real, abarcando todas las instancias posibles de interacción de objetos virtuales y reales.

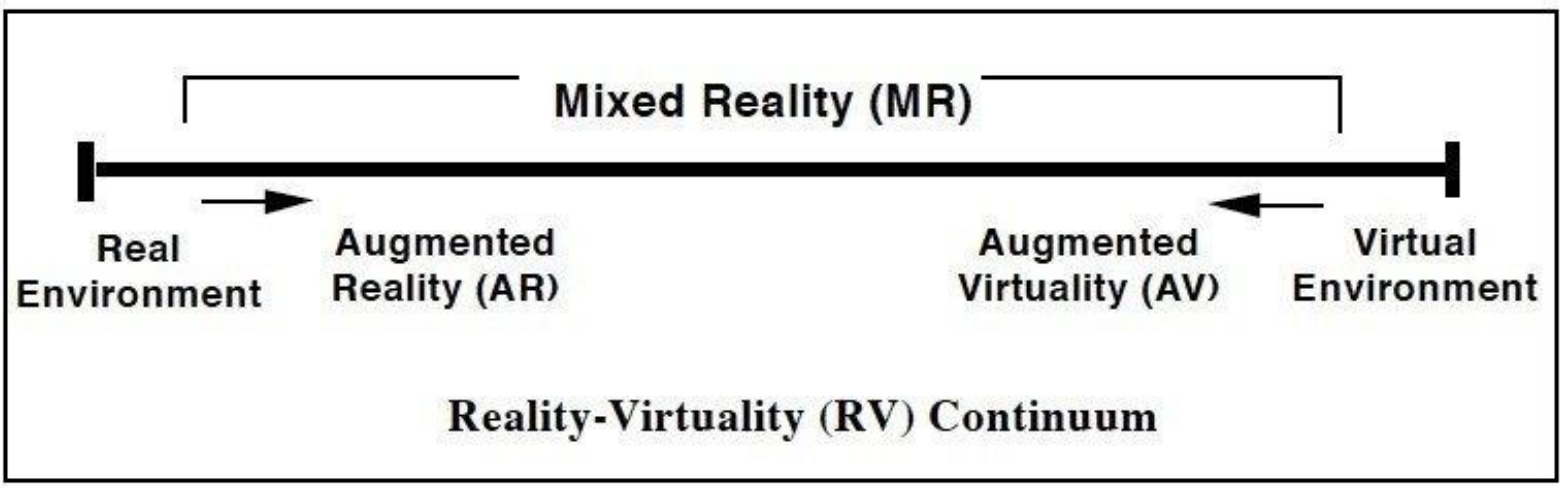

Figura 1. Diagrama de Continuidad Realidad-Virtualidad (Milgram \& Kishino, 1994).

\subsection{Seguimiento y Registro}

La RA necesita seguimiento para superponer contenido virtual sobre vistas del entorno real; es necesario registrar sin problemas el contenido virtual y las vistas del mundo real en tiempo real y corregir la perspectiva para mejorar la presencia de los usuarios en el entorno virtual real. Como se sostiene en (Bekele, Pierdicca, Frontoni, Malinverni, \& Gain, 2018), existe una amplia variedad de técnicas de seguimiento que se basan en cámaras o en sensores físicos.

\section{Seguimiento Basado en Cámaras.}

- Seguimiento basado en marcadores: Utiliza una cámara digital, algoritmos de visión y puntos de referencia reconocibles colocados en entornos interiores o exteriores: estos marcadores pueden ser pasivos (marcadores impresos) o activos (emisor de infrarrojos).

- Seguimiento sin marcadores. Es un seguimiento basado en la visión, generalmente, rastrea la pose de la cámara mediante la detección y el reconocimiento de características geométricas en el entorno real para establecer las imágenes del mundo 3D y $2 \mathrm{D}$ en las coordenadas correspondientes. Este seguimiento depende de elementos geométricos con características distinguibles, como esquinas y bordes de edificios.

- Seguimiento de infrarrojos. El seguimiento de infrarrojos ópticos (IR) es un método para estimar en tiempo real la pose de un objetivo determinado mediante el seguimiento de la posición y la orientación de los marcadores IR activos o pasivos. Los marcadores activos son diodos emisores de infrarrojos que emiten luz infrarroja periódicamente, mientras que los marcadores pasivos consisten en materiales retrorreflectantes que reflejan las luces IR entrantes hacia la fuente.

\section{Seguimiento Basado en Sensores.}

- Seguimiento electromagnético. Se basa en medir la intensidad del campo magnético entre una estación base y un punto de medición, en varias direcciones y orientaciones. La fuerza 
del campo magnético determina la distancia y el cambio de distribución a lo largo de varios ejes, provocados por la rotación del punto de medición, determinan la orientación.

- Seguimiento acústico: Estima la pose de un punto de vista calculando el tiempo necesario para que las ondas de sonido ultrasónicas viajen desde un objetivo emisor a un sensor, que generalmente se mantiene estable en el espacio de seguimiento. Los emisores ultrasónicos están conectados al HMD (Head-Mounted-Displays) y a los dispositivos de interacción, si ambos se están rastreando el punto de vista y las interacciones.

- Seguimiento inercial: Es un sistema de navegación que utiliza giroscopios y acelerómetros para medir la rotación y el movimiento de un objetivo dado, lo que permite el cálculo de pose y velocidad. El acelerómetro mide la aceleración lineal para calcular la posición de un objetivo en relación con algún punto inicial. El giroscopio, por otro lado, mide la velocidad angular para calcular el ángulo rotación de un objetivo en relación con alguna orientación inicial. Por tanto, la pose de un objetivo es la integración de las mediciones del acelerómetro y el giroscopio.

\section{Seguimiento híbrido.}

Es una fusión de los métodos de seguimiento antes mencionados que puede producir mejores resultados que cuando cada uno de ellos se emplea por separado.

\subsection{Modelado de los Entornos Virtuales}

Es el proceso de simulación de objetos reales y su estado en un espacio digital, las reglas de comportamiento que obedecen los objetos y las relaciones e interacciones entre ellos. Desde una perspectiva de adquisición de datos, en (Zhao, 2009) se describen los siguientes tipos de modelo de datos:

- Medición real: La medición real se refiere a los modelos de datos adquiridos a través de los procesos de escaneo 2D y $3 \mathrm{D}$, y cualquier otro proceso que implique el uso de equipos de captura de datos.

- Medición matemática: Se refiere al uso de modelos matemáticos, abstracciones y análisis de métodos experimentales para generar modelo de datos del entorno real.

- Construcción artificial: Se refiere a los modelos de datos generados por la imaginación humana, donde el mundo representado por los modelos de datos es completamente ficticio.

Por su parte, los métodos de modelado se pueden clasificar de acuerdo a las modalidades de percepción del usuario previsto y a los objetos simulados en el entorno de realidad virtual. Desde la perspectiva de percepción sensorial, los métodos de modelado se clasifican en visual, auditivo y háptico. Y desde la perspectiva del objeto simulado, los métodos de modelado se clasifican en apariencia de la escena, comportamiento basado en la física y modelado combinado real-virtual.

\subsection{Dispositivos}

Los elementos principales que forman un sistema de RA son: 
1. Dispositivos de presentación, que se clasifican según el tipo de contenido virtual que muestran, el más común es el visual.

- Head-Mounted-Displays (HMD): Son dispositivos de visualización parecidos a un casco; pueden ser ópticos o de video transparente. Los ópticos transparentes se superponen al espacio real para mostrar la vista aumentada: los usuarios ven el contenido sintético y el entorno real coexistiendo en un espacio virtual. En el caso de los videos transparentes, un dispositivo informático procesa las imágenes que llegan a través de las cámaras montadas en el HMD, aumentando la escena con información virtual y renderizando la mezcla de imágenes.

- Realidad Aumentada Espacial: Se basa en proyectores digitales para mostrar información gráfica sobre los objetos físicos. En este caso, la pantalla está separada de los usuarios del sistema y dado que la pantalla no está asociada a cada usuario permite a grupos de usuarios utilizarlo al mimo tiempo.

- Portátil: Son dispositivos manuales que incorporan una pantalla que cabe en la mano del usuario, como tabletas o móviles, y sobre la cual se muestra la información virtual añadida a la real. Utiliza enfoques de video transparente para superponer contenido virtual sobre vistas del entorno real.

2. Dispositivos de seguimiento, entre los que se encuentran cámaras digitales, acelerómetros, giroscopios, brújula, sensores inalámbricos, GPS, entre otros.

3. Dispositivos de entrada, que permiten que el usuario transmita sus deseos al sistema mediante controles físicos, controles del habla o control por gestos. Los controles del habla incluyen sistemas de reconocimiento de voz que traducen las palabras del usuario en instrucciones de computadora; mientras que por medio del control de gestos se pueden interpretar los movimientos del cuerpo del usuario mediante la detección visual o mediante sensores integrados en un dispositivo periférico, como un lápiz, puntero, guante, pulseras inalámbricas, entre otros.

4. Computadora, como elemento de procesamiento, que es capaz de interpretar la información del mundo real que recibe el usuario, generar la información virtual que cada servicio concreto necesite y mezclarla de forma adecuada. También se incluyen a dispositivos móviles, como teléfonos inteligentes y tabletas, como elementos de procesamiento.

\subsection{Herramientas de Desarrollo}

Las herramientas utilizadas para el desarrollo de aplicaciones de RA, conocidas como Kits de Desarrollo de Software (SDK), proporcionan un entorno donde los usuarios programan funcionalidades que compondrán aplicaciones de software con las capacidades y recursos de la RA.

En el mercado es posible encontrar distintas alternativas de SDK para RA. Algunos estudios, como (Herpich, Guarese, \& Tarouco, 2017), (Fierro, Manosalvas, \& Hidrobo, 2018), (Jooste, Rautenbach, \& Coetzee, 2015) presentan comparaciones para facilitar a los desarrolladores la elección del SDK. Entre los criterios que se definen para la comparación, se encuentran: 
- Plataforma: sistemas operativos móviles que soportan la RA.

- Sistema de Posicionamiento Global (GPS): utilización de tecnología GPS, etiquetas, actualización de puntos geográficos y recuperación de datos.

- Imágenes: posibilidad de reproducir un video y generar imágenes en 2D y 3D.

- Documentación: existencia de información de soporte para instalación y desarrollo de aplicaciones.

- Framework: disponibilidad de un entorno propio para generar imágenes, modelos 3D y videos.

- Licencia: Disponibilidad para usar la herramienta ya sea pagando una licencia, por medio de una versión de prueba o es totalmente gratis.

- Integración: Facilidad para integrar con otras aplicaciones. Por ejemplo con Unity ${ }^{1}$, que si bien fue desarrollada para la creación de videojuegos multiplataforma, también permite añadir elementos de RA, imágenes en 2D y 3D renderizadas en tiempo real y minijuegos.

En la Tabla 1, mostramos un resumen de las herramientas analizadas:

- ARToolKit ${ }^{2}$ : Permite la superposición de imágenes virtuales en el mundo real, teniendo en cuenta el punto de vista de los usuarios, calculando para esto la posición y orientación real de la cámara del smartphone en relación con los marcadores físicos, que pueden ser cualquier patrón cuadrado. Al haber sufrido falta de actividad por parte de la comunidad, en 2017 se creó "artoolkitX" que cuenta con respaldo de una empresa china, para poder mantener y permitir que el software se desarrolle.

- ARCore ${ }^{3}$ : Es la Plataforma de Google para crear RA. Es capaz de rastrear la posición del 6martphone a medida que se mueve y desarrollar su propia comprensión del mundo real. Puede reconocer superficies planas e imágenes personalizadas. Tiene tres características importantes: "Motion tracking" que permite comprender donde está el smartphone en relación con el mundo que lo rodea, "Environmental understanding" que permite detectar superficies planas y "Light estimation" que permite iluminar los objetos virtuales de acuerdo con la iluminación del entorno.

- EasyAR Sense ${ }^{4}:$ Al igual que el resto de las herramientas ya mencionadas Easy Ar Sense posee la capacidad de detectar el mundo real y agregar objetos virtuales de distintas formas mediante la utilización de una cámara de smartphone. Entre las características más importantes que posee se encuentran:

- Sparse SpatialMap: es quien permite escanear el entorno construyendo un mapa 3D del mundo físico y agregarle objetos virtuales persistentes en el entorno. Se puede cargar varios mapas, guardar y compartir los mapas 3D entre diferentes dispositivos, permitiendo que cuando otras personas visiten ese mismo espacio se comparen las imágenes tomadas con la cámara del smartphone y el mapa 3D, si coinciden se permite visualizar los objetos virtuales previamente agregados.

- Dense SpatialMap: permite escanear el entorno construyendo una malla 3D formada por vértices y caras sobre la superficie del entorno, lo que genera el agregado de colisión y oclusión en los objetos virtuales, permitiendo que estos interactúen con el mundo físico sin problemas.

\footnotetext{
${ }^{1}$ https://unity.com/es

2 http://www.artoolkitx.org

${ }^{3}$ https://developers.google.com/ar

${ }^{4}$ https://www.easyar.com/
} 
- Motion Tracking: hace referencia al seguimiento de la posición del dispositivo en relación con el mundo físico. Hace uso de la técnica denominada Visual-Inertial Simultaneous Localization and Mapping (VISLAM), que reconoce características sobresalientes en la imagen de la cámara y rastrea las diferentes posiciones de los cuadros continuos fusionando toda la información con datos IMU. Por si solo no admite características importantes por lo que es necesario usarlo en conjunto con Sparse SpatialMap, Dense SpatialMap, etc.

- Surface Tracking: similar al Motion Tracking hace seguimiento de la posición del dispositivo, pero con relación a superficies. Un objeto virtual siempre se coloca en un punto específico en determinada superficie la cual se actualiza cada vez que se mueve el dispositivo.

- 3D Object Tracking: detecta y rastrea objetos 3D ricos en textura del mundo físico. Se utiliza modelos 3D virtuales que sean iguales a los objetos del mundo físico y se agrega contenido virtual.

- Planar Image Tracking: permite reconocer y rastrear imágenes planas ricas en textura del mundo físico. Utiliza modelos 3D virtuales que sean iguales a las imágenes del mundo físico y se les agrega contenido virtual. Para realizar la aplicación se utilizó la herramienta Planar Image Tracking en conjunto con Motion Tracking.

- Vuforia ${ }^{5}$ : El reconocimiento del mundo real que hace, se centra, fundamentalmente, en la cámara del smartphone y en reconocer lo que éste enfoca. Actualmente, es capaz de reconocer: códigos bidimensionales, textos, objetos cilíndricos, objetos construidos a partir de superficies planas, e imágenes personalizadas. Tiene una característica llamada Smart Terrain ${ }^{\mathrm{TM}}$, que permite que el móvil sea capaz de reconocer una superficie y, además, reconocer e integrar objetos reales en la parte virtual.

Tabla 1. SDK de RA analizados.

\begin{tabular}{|l|l|l|l|l|}
\hline & ARToolKit & ARCore & EasyAR Sense & Vuforia \\
\hline Versión & & $\mathbf{1 . 1 6 . 0}$ & $\mathbf{4 . 0 . 0}$ & \\
\hline Plataformas & Android & Android & $\begin{array}{l}\text { Android } \\
\text { IOS }\end{array}$ & $\begin{array}{l}\text { Android } \\
\text { IOS }\end{array}$ \\
\hline GPS & $\mathrm{No}$ & $\mathrm{No}$ & $\mathrm{No}$ & $\mathrm{Si}$ \\
\hline Imágenes & $\mathrm{Si}$ & $\mathrm{Si}$ & $\mathrm{Si}$ & $\mathrm{Si}$ \\
\hline Documentación & $\mathrm{Si}$ & $\mathrm{Si}$ & $\mathrm{Si}$ & $\mathrm{Si}$ \\
\hline Framework & $\mathrm{No}$ & $\mathrm{No}$ & $\mathrm{No}$ & No \\
\hline Licencias & Gratis & Gratis & Gratis & Gratis \\
& Comercial & & Comercial & Comercial \\
\hline Integración Unity & $\mathrm{Si}$ & $\mathrm{Si}$ & $\mathrm{Si}$ & $\mathrm{Si}$ \\
\hline
\end{tabular}

Si bien todos los SDK analizados permiten generar contenido de RA, sólo EasyAR Sense permite reconocer múltiples objetivos simultáneamente, generar un código único para la aplicación desarrollada y ejecutarla en smarthphones de capacidad limitada.

${ }^{5}$ https://developer.vuforia.com/ 


\section{TURISMO CULTURAL CON RA}

De acuerdo con la UNESCO, "el patrimonio es la herencia cultural propia del pasado de una comunidad, mantenida hasta la actualidad y transmitida a las generaciones presentes". Uno de los pilares de la gestión del patrimonio es la difusión; y en este sentido, las nuevas tecnologías han mejorado el acercamiento de los usuarios al Patrimonio Cultural.

Tal como se sostiene en (Torres, 2013), "las posibilidades que ofrece la tecnología de realidad aumentada en espacios expositivos y patrimoniales han crecido exponencialmente en los últimos años debido a su gran atractivo para el público, constituyendo un importante recurso dentro de sus programas museográficos más vanguardistas. El uso de RA en el campo del Patrimonio Cultural ha demostrado su utilidad para la difusión y el conocimiento de objetos culturales; por ejemplo, en la restauración virtual de objetos del patrimonio religioso, el diseño de historias interactivas para visitantes de sitios culturales, el desarrollo de aplicaciones de turismo móvil, y la construcción de sistemas para visualizar reconstrucciones de edificios antiguos.

En este sentido, la RA puede desempeñar un papel importante en la herencia cultural, ya que permite la visualización simultánea de datos 3D virtuales y reales. El modelado virtual y la reconstrucción 3D son herramientas muy utilizadas para recrear, analizar y visualizar tanto sitios arqueológicos o edificios, como esculturas. Para esto, deben tenerse en cuenta algunos principios de diseño, como: 1) alta precisión geométrica, 2) alto nivel de captura de automatización para todos los detalles, 3) bajo costo, 4) fotorrealismo, 5) flexibilidad, 6) portabilidad y 7) eficiencia del tamaño del modelo (El-Hakim, Beraldin, Picard, \& Godin, 2004).

De acuerdo a lo planteado en (Attila \& Edit, 2012), las principales ventajas de usar RA en sitios de Patrimonio Cultural son:

1. El contenido aumentado relacionado con un objeto o atracción determinados se puede incluir en una forma que no daña ni perturba su entorno. Por ejemplo, no es necesario paneles de información, puntos de información, etc.

2. Los paneles de información requieren espacio en una sala de exhibición o pasillo y por la cantidad de exhibiciones la información se limita al tamaño del tablero. Con una base de datos virtual obtenemos mucha más información, escrita o en audio y en innumerables idiomas, y podemos exponer más objetos en un espacio gratuito.

3. El contenido aumentado mejora la valoración del cliente, lo que amplía el valor del servicio, ayuda a la satisfacción del cliente, genera un boca a boca positivo y en general resultados más altos de asistencia.

4. Brinda la oportunidad de compartir la experiencia del cliente en tiempo real a través de comunidades en línea mediante redes sociales, lo que también puede generar una mayor asistencia y conocimiento.

\subsection{Aplicaciones Móviles}

A partir de un análisis exploratorio de la literatura es posible identificar aplicaciones de Realidad Aumentada utilizadas en el dominio de Turismo Cultural: 
- Archeoguide (Vlahakis, y otros, 2002), uno de los primeros sistemas móviles que ofrece una guía electrónica personalizada de sitios arqueológicos al aire libre, con el objetivo de ayudar a los usuarios a navegar y aprovechar al máximo su visita, y permitir la recopilación, explotación y actualización de datos arqueológicos en cualquier sitio dado. Aprovecha la RA, la visualización en 3D, y las redes para presentar a los usuarios recorridos en el sitio y en línea del sitio físico de acuerdo con un perfil y comportamiento durante el recorrido.

- PRISMA (Fritz, Susperregui, \& Linaza, 2005), una aplicación de RA para patrimonio cultural que por medio de la incorporación de los prismáticos Tourist AR, muestra contenido aumentado y permite la interacción con un menú emergente para cambiar entre diferentes tipos de contenido.

- Lifeplus (Vlahakis, Pliakas, Demiris, \& Ioannidis, 2003), esta experiencia de RA se basaba en insertar, dentro del escenario real de los restos de Pompeya, animaciones virtuales de flora y fauna y recreaciones de escenas de la vida cotidiana mediante gráficos y actores virtuales en 3D. Mediante el uso de gafas de RA, un sistema inalámbrico determinaba los gráficos 3D que se insertaban en el entorno real y mostraba la imagen aumentada en el display de las gafas, junto a sonidos o narración relacionados.

- UrbanMix (Portalés, Lerma, \& Navarro, 2010), una aplicación de RA que consistió en aumentar el entorno actual con dos edificios del centro histórico de Valencia: las Torres de Serranos y el Miguelete, recreando un escenario combinado de dos espacios urbanísticos que se encuentran distantes espacial y temporalmente.

- MixAR (Narciso, Pádua, Adão, Peres, \& Magalhães, 2015) es un sistema que mediante RA permite visualizar edificios virtuales, alineados correctamente con estructuras del mundo real, según la ubicación del usuario, durante la navegación al aire libre.

- Fuendetodos (Conde, 2020), es una aplicación turística para móviles que incorpora RA, geolocalización y gamificación, para ofrecer información sobre el municipio, permitir sacarse una foto junto a un retrato de Francisco de Goya en la casa natal del pintor o mostrar en 3D uno de los asnos que aparecen en la serie de Los Caprichos de Goya.

También en la tienda de descargas Google Play se pueden encontrar algunos proyectos que involucran RA en aplicaciones turísticas (Luna, Etxeberria, \& Gracia, 2019); entre ellas:

- Guideo ${ }^{6}$ es una aplicación móvil gratuita, que estuvo actualizada hasta 2016 y que ofrece realizar rutas turísticas, culturales y temáticas por diversos lugares de interés.

- Castellones de Ceal $\mathrm{RA}^{7}$ ofrece información sobre este yacimiento arqueológico, y por medio de RA, permite escanear códigos que se encuentran dentro del Museo Ibero de Jaén, para ver la reconstrucción de tumbas funerarias ibéricas en tres dimensiones.

- La Alhambra, Castillo Rojo ${ }^{8}$ es una app orientada al público infantil, donde se ofrece información del lugar mediante audio, imágenes y guías virtuales que interpretan el patrimonio ante el que se encuentra el visitante. Se plantea como un juego para encontrar

\footnotetext{
${ }^{6}$ https://play.google.com/store/apps/details?id=com.bookguideo\&hl=es_AR

${ }^{7}$ https://play.google.com/store/apps/details?id=com.esTRESd.Castellones\&hl=es

${ }^{8}$ https://play.google.com/store/apps/details?id=com.global3d.acr\&hl=es_AR
} 
las llaves escondidas mediante la activación de la cámara del móvil. Durante el recorrido se encuentran códigos que permiten utilizar la RA y acceder a más contenido.

- Turismo San Luis $4.0^{9}$ ofrece, al enfocar el celular sobre folletos o edificios turísticos identificados con el ícono de AR+, la posibilidad de acceder a videos, música autóctona e información sobre servicios del lugar.

- La Piedad ${ }^{10}$ es una guía virtual de contenido multimedia digital con RA sobre 11 lugares del cementerio La Piedad en Misiones, Argentina. Muestra una animación de personajes históricos que cuentan su propia historia. No requiere celular de última generación ni conexión WiFi.

- Waia ${ }^{11}$ ofrece un recorrido por la ciudad de Ushuaia utilizando RA. Para poder utilizarla, se apunta el celular al código QR que figura en tótems instalados en la ciudad, pudiendo acceder a datos históricos sobre edificios principales y su historia.

A partir de las aplicaciones identificadas, se puede comprobar que, tal como se propone en (Bekele, Pierdicca, Frontoni, Malinverni, \& Gain, 2018), las principales características funcionales de las aplicaciones de RA para Turismo Cultural se pueden clasificar en:

- Educación: tiene como objetivo permitir a los usuarios aprender los aspectos históricos del Patrimonio Cultural tangible e intangible.

- Exposición: tiene como objetivo mejorar la experiencia del visitante en museos físicos y sitios patrimoniales, generalmente a través de la guía turística.

- Exploración: ayuda a los usuarios a visualizar y explorar puntos de vista históricos y actuales, para descubrir, interpretar y adquirir nuevos conocimientos.

- Reconstrucción: tiene como objetivo permitir a los usuarios visualizar e interactuar con vistas históricas reconstruidas.

- Museos virtuales: simulan y presentan al público el patrimonio cultural en forma de museo digital.

\section{MATERIALES Y MÉTODOS}

El objetivo principal de esta investigación fue desarrollar guías de diseño de UX que sean aplicables a aplicaciones móviles para Turismo Cultura con RA. Para desarrollar estas guías, se planteó un proceso basado en la propuesta de (Quiñones, Rusu, \& Rusu, 2018). A continuación se describe en detalle cada una de las actividades que lo conforman.

\subsection{Revisión}

Se realiza una exploración de la literatura para identificar características de UX relacionadas con RA y turismo. La revisión de la literatura permitió identificar características particulares de UX relacionados con RA y turismo. Si bien el turismo es un área altamente aplicable para la RA, está poco explorada en cuanto a estudios de UX (Dey, Billinghurst, Lindeman, \& Swan, 2018). Los resultados de la revisión realizada se resumen en la Tabla 2.

\footnotetext{
${ }^{9}$ https://play.google.com/store/apps/details?id=com.grupocentro.TurismoSanLuis

${ }^{10}$ https://play.google.com/store/apps/details?id=com.CCUNaM.LaPiedad

${ }^{11}$ https://play.google.com/store/apps/details?id=com.UNTDF.WAIA
} 
Tabla 2. Características de UX en aplicaciones turísticas con RA.

\begin{tabular}{|c|c|}
\hline Referencia & Características de UX \\
\hline $\begin{array}{l}\text { (Boboc, Duguleană, Voinea, } \\
\text { Postelnicu, Popovici, \& Carrozzino, } \\
\text { 2019) }\end{array}$ & $\begin{array}{l}\text { Comprensibilidad, "manipulabilidad", disfrute y } \\
\text { utilidad. }\end{array}$ \\
\hline (Han \& Jung, 2018) & $\begin{array}{l}\text { Simplicidad, filtrado de información, función social, } \\
\text { privacidad y seguridad, navegación, lenguaje, calidad } \\
\text { de la información y accesibilidad. }\end{array}$ \\
\hline (Sylaiou, y otros, 2015) & $\begin{array}{l}\text { Funcionalidad de elementos gráficos, calidad de } \\
\text { modelos VRML), navegación, ayuda, funcionalidad } \\
\text { de elementos de RA, estética, contenido enriquecido, } \\
\text { aspectos tecnológicos. }\end{array}$ \\
\hline (Dahlan, 2016) & $\begin{array}{l}\text { Interfaz de usuario simple, reconocimiento de } \\
\text { ubicación, velocidad, asequibilidad, privacidad y } \\
\text { seguridad, accesibilidad, personalización y redes } \\
\text { sociales, eficiente, fácil de usar. }\end{array}$ \\
\hline $\begin{array}{l}\text { (Lim, Selamat, Alias, Krejcar, \& } \\
\text { Fujita, 2019) }\end{array}$ & $\begin{array}{l}\text { Usabilidad/ experiencia, facilidad de aprendizaje, } \\
\text { contenido, motivación, compromiso, adaptación, } \\
\text { satisfacción, comportamiento, efectividad, eficiencia, } \\
\text { facilidad de uso, emoción, diversión, carga cognitiva, } \\
\text { preferencias, diseño de interface, seguridad, otras. }\end{array}$ \\
\hline (Han, Tom Dieck, \& Jung, 2018) & $\begin{array}{l}\text { Contenido, presentación, funcionalidad, interacción, } \\
\text { atributos pragmáticos, atributos hedónicos. }\end{array}$ \\
\hline
\end{tabular}

\subsection{Agrupación}

Se agrupan las características de UX identificadas, eliminando características repetidas y combinando aquellas que están relacionadas. La agrupación propuesta se basa en las categorías detalladas en la Tabla 3.

Luego, las recomendaciones identificadas en la revisión se vuelcan en una planilla, asociadas con alguna de estas categorías. 
Tabla 3. Categorización de Características de UX en aplicaciones de RA para turismo.

\begin{tabular}{|l|l|}
\hline Categoría & Descripción \\
\hline Usabilidad & $\begin{array}{l}\text { Capacidad de la aplicación de ser usada y manipulada por los } \\
\text { usuarios bajo condiciones específicas. }\end{array}$ \\
\hline Contenido & $\begin{array}{l}\text { Aspectos relacionados con la relevancia y precisión de la } \\
\text { información presentada por la aplicación. }\end{array}$ \\
\hline Disfrute & $\begin{array}{l}\text { Grado en que un usuario obtiene placer al satisfacer sus } \\
\text { necesidades personales. }\end{array}$ \\
\hline Comprensibilidad & $\begin{array}{l}\text { Capacidad de la aplicación de permitir al usuario comprender si } \\
\text { la aplicación es adecuada y cómo puede ser utilizada para tareas } \\
\text { específicas. }\end{array}$ \\
\hline Atracción & $\begin{array}{l}\text { Aspectos relacionados con la atracción del diseño estético de la } \\
\text { interfaz de la aplicación. }\end{array}$ \\
\hline Funcionalidad & $\begin{array}{l}\text { Capacidad de la aplicación para brindar funciones que satisfacen } \\
\text { las necesidades declaradas e implícitas, cuando se usa en las } \\
\text { condiciones especificadas. Se incluye funcionalidad espećfica de } \\
\text { Realidad Aumentada, compartir contenido en redes sociales, y } \\
\text { cambiar el idioma. }\end{array}$ \\
\hline Privacidad y seguridad & $\begin{array}{l}\text { Capacidad de protección de la información y los datos de manera } \\
\text { que personas o sistemas no autorizados no puedan leerlos o } \\
\text { modificarlos. }\end{array}$ \\
\hline Eticiencia & $\begin{array}{l}\text { Aspectos relacionados con la navegación por las pantallas de la } \\
\text { aplicación. }\end{array}$ \\
\hline Precisión & $\begin{array}{l}\text { Capacidad de la aplicación que permite que sea utilizada por } \\
\text { usuarios con determinadas características y discapacidades. }\end{array}$ \\
\hline $\begin{array}{l}\text { Aspectos relacionados con el desempeño relativo a la cantidad de } \\
\text { recursos utilizados bajo determinadas condiciones. }\end{array}$ \\
\hline Aspectos relacionados con el propósito general de la aplicación. \\
\hline $\begin{array}{l}\text { Capacidad de la aplicación para proveer resultados correctos con } \\
\text { el nivel de precisión requerido. }\end{array}$ \\
\hline Ecciliad
\end{tabular}

\subsection{Selección}

La lista de recomendaciones agrupadas se analiza, determinando si conservarla, eliminarla o adaptarla. Se crea un conjunto preliminar de guías de diseño UX.

La selección del conjunto de guías se basa en el análisis de expertos en UX; para esto, se entrega a cada experto la planilla con la lista de recomendaciones agrupadas en categorías. Sobre esa planilla, cada experto indica en la columna "Acción", si esa recomendación debe conservarse, eliminarse o modificarse. En caso de indicar modificaciones, el experto describe, en la columna "Modificación propuesta", el cambio sugerido sobre redacción o sobre categoría. En la columna "Observaciones", puede agregar cualquier comentario pertinente que no pudiera reflejar en las otras columnas; por ejemplo, que no entiende lo que expresa esa recomendación. 


\section{RESULTADOS}

En la etapa de Revisión se recopilaron 360 recomendaciones, que se agruparon en 12 categorías, y se propuso su análisis a 4 expertos en UX.

Como resultado de este análisis, en primer lugar, surgió la propuesta de modificar las categorías definidas en la Tabla 3, entre ellas:

1. Eliminar todas las características de las categorías "Disfrute" y "Utilidad" (propuesta de Experto1).

2. Modificar los nombres de las categorías, asociándolos con características del modelo ISO/IEC 25010 (ISO/IEC JTC 1/SC 7, 2011) (propuesta de Experto1).

3. Conservar la categoría "Contenido", aún cuando no se asocia directamente con alguna de las características del modelo ISO/IEC 25010, (propuesta de Experto4).

4. Incluir las categorías "Comprensibilidad", "Accesibilidad", y "Estética" en "Usabilidad", (propuesta de Experto2 y Experto3).

5. Incluir la categoría "Navegación" en "Pertinencia Funcional", (propuesta de Experto2).

En la Tabla 4 se resumen los cambios consensuados por los 4 expertos; resultando en 312 recomendaciones agrupadas en 6 categorías, para su posterior análisis.

Tabla 4. Modificación de Categorización de Características de UX.

\begin{tabular}{|l|l|r|}
\hline Categoría Original & Categoría Nueva & Recomendaciones \\
\hline Usabilidad & Usabilidad & 113 \\
\hline Contenido & Contenido & 52 \\
\hline Disfrute & (Se elimina) & No corresponde \\
\hline Comprensibilidad & (Se incluye en Usabilidad) & No corresponde \\
\hline Atracción & (Se incluye en Usabilidad) & No corresponde \\
\hline Funcionalidad & Pertinencia funcional & 88 \\
\hline Privacidad y seguridad & Seguridad & 11 \\
\hline Navegación & (Se incluye en Pertinencia funcional) & No corresponde \\
\hline Accesibilidad & (Se incluye en Usabilidad) & No corresponde \\
\hline Eficiencia & Eficiencia & 28 \\
\hline Utilidad & (Se elimina) & No corresponde \\
\hline Precisión & Correctitud & 20 \\
\hline \multicolumn{2}{r}{ Total } & 312 \\
\hline
\end{tabular}

En la Tabla 5, se muestra el conjunto preliminar de 52 guías de UX seleccionado. Las guías que se incluyen en este conjunto preliminar son aquellas que recibieron mayor aceptación por parte de los expertos evaluadores. Es decir, aquellas guías que recibieron la recomendación de "conservar" por parte de la mayoría de los expertos; en este caso, de por lo menos 3 expertos. Por sugerencia de los expertos, también se reescribió cada guía formulando oraciones declarativas en lugar de oraciones interrogativas. 
Tabla 5. Conjunto de guías de UX para aplicaciones turísticas con RA.

\begin{tabular}{|c|c|c|c|c|c|c|}
\hline Categoría & Guías &  & 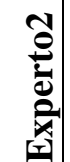 & 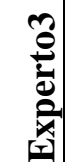 & 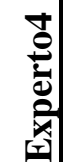 & $\stackrel{\pi}{\theta}$ \\
\hline \multirow[t]{15}{*}{ Usabilidad } & $\begin{array}{l}\text { USA1. Brindar interacción que resulte cómoda para brazos y } \\
\text { manos. }\end{array}$ & $\sqrt{ }$ & $\sqrt{ }$ & & $\checkmark$ & 3 \\
\hline & $\begin{array}{l}\text { USA2. Estandarizar la ubicación de enlaces y menús en toda } \\
\text { la aplicación. }\end{array}$ & $\checkmark$ & $\checkmark$ & $\checkmark$ & $\checkmark$ & 4 \\
\hline & $\begin{array}{l}\text { USA3. Ofrecer mensajes útiles cuando el usuario no sabe } \\
\text { cómo proceder. }\end{array}$ & $\checkmark$ & $\checkmark$ & $\checkmark$ & $\checkmark$ & 4 \\
\hline & $\begin{array}{l}\text { USA4. Facilitar la interacción en pantallas de tamaño } \\
\text { reducido. }\end{array}$ & $\checkmark$ & $\checkmark$ & $\checkmark$ & & 3 \\
\hline & $\begin{array}{l}\text { USA5. Evitar que el usuario tenga que memorizar cosas } \\
\text { innecesariamente. }\end{array}$ & $\checkmark$ & $\checkmark$ & $\checkmark$ & $\checkmark$ & 4 \\
\hline & USA6. Utilizar contrastes adecuados entre imágenes y fondo. & $\checkmark$ & $\checkmark$ & $\checkmark$ & $\checkmark$ & 4 \\
\hline & $\begin{array}{l}\text { USA7. Utilizar tipografías y colores que se ajusten a la } \\
\text { aplicación. }\end{array}$ & $\checkmark$ & $\checkmark$ & $\checkmark$ & & 3 \\
\hline & USA8. Utilizar iconos consistentes y familiares. & $\checkmark$ & $\checkmark$ & $\checkmark$ & $\checkmark$ & 4 \\
\hline & $\begin{array}{l}\text { USA9. Agrupar elementos de la interface de usuario en zonas } \\
\text { lógicas. }\end{array}$ & $\checkmark$ & $\checkmark$ & $\checkmark$ & $\checkmark$ & 4 \\
\hline & $\begin{array}{l}\text { USA10. Espaciar los elementos gráficos para hacer clic } \\
\text { fácilmente. }\end{array}$ & $\checkmark$ & $\checkmark$ & $\checkmark$ & $\checkmark$ & 4 \\
\hline & USA11. Asegurar la mejor calidad posible de imágenes. & $\checkmark$ & $\checkmark$ & $\checkmark$ & $\checkmark$ & 4 \\
\hline & USA12. Aplicar un estilo de diseño gráfico homogéneo. & $\checkmark$ & $\checkmark$ & $\checkmark$ & $\checkmark$ & 4 \\
\hline & $\begin{array}{l}\text { USA13. Reducir al máximo los pasos para completar las } \\
\text { tareas del usuario. }\end{array}$ & $\checkmark$ & $\checkmark$ & $\checkmark$ & $\checkmark$ & 4 \\
\hline & USA14. Crear opciones de menú lógicas y distintivas. & $\checkmark$ & $\checkmark$ & $\checkmark$ & $\checkmark$ & 4 \\
\hline & $\begin{array}{l}\text { USA15. Visualizar claramente el estado del proceso en } \\
\text { ejecución. }\end{array}$ & $\checkmark$ & $\checkmark$ & & $\checkmark$ & 3 \\
\hline \multirow[t]{12}{*}{ Contenido } & CON1. Proporcionar información científicamente precisa. & $\checkmark$ & & $\checkmark$ & $\checkmark$ & 3 \\
\hline & CON2. Incluir textos claros y simbología intuitiva. & $\checkmark$ & $\checkmark$ & $\checkmark$ & $\checkmark$ & 4 \\
\hline & CON3. Identificar contenidos según título, fuente y fecha. & $\checkmark$ & $\checkmark$ & $\checkmark$ & $\checkmark$ & 4 \\
\hline & CON4. Ofrecer contenido actualizado. & $\checkmark$ & $\checkmark$ & $\checkmark$ & $\checkmark$ & 4 \\
\hline & CON5. Ofrecer contenido organizado. & $\checkmark$ & $\checkmark$ & $\checkmark$ & $\checkmark$ & 4 \\
\hline & CON6. Incluir información relevante y completa. & $\checkmark$ & $\checkmark$ & $\checkmark$ & $\checkmark$ & 4 \\
\hline & CON7. No incluir información repetitiva. & $\checkmark$ & & $\checkmark$ & $\checkmark$ & 3 \\
\hline & $\begin{array}{l}\text { CON8. Asegurar que el contenido aumentado se relaciona con } \\
\text { la realidad. }\end{array}$ & $\checkmark$ & $\checkmark$ & $\checkmark$ & $\checkmark$ & 4 \\
\hline & CON9. Asegurar ortografía y gramática correctas. & $\checkmark$ & $\checkmark$ & $\checkmark$ & $\checkmark$ & 4 \\
\hline & $\begin{array}{l}\text { CON10. Incluir contenido audiovisual como apoyo al } \\
\text { contenido textual. }\end{array}$ & $\checkmark$ & $\checkmark$ & $\checkmark$ & $\checkmark$ & 4 \\
\hline & $\begin{array}{l}\text { CON11. Escribir títulos breves, pero suficientes para } \\
\text { comunicar. }\end{array}$ & & $\checkmark$ & $\checkmark$ & $\checkmark$ & 3 \\
\hline & CON12. Utilizar lenguaje claro para usuarios sin experiencia. & $\checkmark$ & & $\checkmark$ & $\checkmark$ & 3 \\
\hline
\end{tabular}


Tabla 5 (continuación). Conjunto de guías de UX para aplicaciones turísticas con RA.

\begin{tabular}{|c|c|c|c|c|c|c|}
\hline Categoría & Guías & 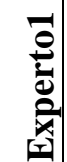 & 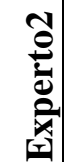 & 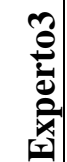 &  & $\stackrel{\bar{\sigma}}{\theta}$ \\
\hline \multirow[t]{11}{*}{$\begin{array}{l}\text { Pertinencia } \\
\text { Funcional }\end{array}$} & $\begin{array}{l}\text { PER1. Permitir compartir y comentar contenido en redes } \\
\text { sociales. }\end{array}$ & $\checkmark$ & $\checkmark$ & $\checkmark$ & $\checkmark$ & 4 \\
\hline & $\begin{array}{l}\text { PER2. Permitir identificar la ubicación, en aplicaciones } \\
\text { basadas en mapas. }\end{array}$ & $\checkmark$ & $\checkmark$ & $\checkmark$ & $\checkmark$ & 4 \\
\hline & PER3. Ofrecer opciones de múltiples idiomas. & $\checkmark$ & $\checkmark$ & $\checkmark$ & $\checkmark$ & 4 \\
\hline & PER4. Permitir navegación usando RA. & $\checkmark$ & $\checkmark$ & $\checkmark$ & $\checkmark$ & 4 \\
\hline & PER5. Incluir canal de contacto para usuarios. & $\checkmark$ & $\checkmark$ & $\checkmark$ & & 3 \\
\hline & PER6. Incluir una opción de salida de la aplicación. & $\checkmark$ & $\checkmark$ & $\checkmark$ & $\checkmark$ & 4 \\
\hline & PER7. Ofrecer comandos de navegación simples e intuitivos. & $\checkmark$ & $\checkmark$ & $\checkmark$ & $\checkmark$ & 4 \\
\hline & PER8. Ofrecer la posibilidad de buscar información. & $\checkmark$ & $\checkmark$ & $\checkmark$ & $\checkmark$ & 4 \\
\hline & $\begin{array}{l}\text { PER9. Ofrecer una forma de entrada adicional al entorno de } \\
\text { RA. }\end{array}$ & $\checkmark$ & $\checkmark$ & $\checkmark$ & $\checkmark$ & 4 \\
\hline & $\begin{array}{l}\text { PER10. Ofrecer explicación sobre el funcionamiento de la } \\
\text { aplicación. }\end{array}$ & $\checkmark$ & $\checkmark$ & $\checkmark$ & $\checkmark$ & 4 \\
\hline & $\begin{array}{l}\text { PER11. Identificar claramente en cuál zona hacer clic y en } \\
\text { cuál no. }\end{array}$ & & $\checkmark$ & $\checkmark$ & $\checkmark$ & 3 \\
\hline \multirow[t]{2}{*}{ Seguridad } & SEG1. Proteger la privacidad de los usuarios. & $\checkmark$ & $\checkmark$ & $\checkmark$ & $\sqrt{ }$ & 4 \\
\hline & SEG2. Incluir certificado de seguridad. & $\checkmark$ & $\checkmark$ & $\checkmark$ & $\checkmark$ & 4 \\
\hline \multirow[t]{6}{*}{ Eficiencia } & EFI1. Permitir el acceso a funcionalidades fuera de línea. & $\checkmark$ & $\checkmark$ & & $\checkmark$ & 3 \\
\hline & EFI2. Permitir el uso de pantalla vertical y horizontal. & $\checkmark$ & $\checkmark$ & $\checkmark$ & $\checkmark$ & 4 \\
\hline & EFI3. Minimizar el peso de archivos gráficos y multimedia. & $\checkmark$ & $\checkmark$ & $\checkmark$ & $\checkmark$ & 4 \\
\hline & $\begin{array}{l}\text { EFI4. Permitir el uso de la aplicación en diferentes } \\
\text { plataformas. }\end{array}$ & $\checkmark$ & $\checkmark$ & $\checkmark$ & $\checkmark$ & 4 \\
\hline & EFI5. Evitar que la aplicación se suspenda. & $\checkmark$ & $\checkmark$ & $\checkmark$ & $\checkmark$ & 4 \\
\hline & $\begin{array}{l}\text { EFI6. Minimizar la velocidad de carga de contenidos de la } \\
\text { aplicación. }\end{array}$ & $\checkmark$ & $\checkmark$ & $\checkmark$ & $\checkmark$ & 4 \\
\hline \multirow[t]{6}{*}{ Correctitud } & $\begin{array}{l}\text { COR1. Presentar los objetos de RA en forma y posición } \\
\text { adecuada. }\end{array}$ & $\checkmark$ & $\checkmark$ & $\checkmark$ & $\checkmark$ & 4 \\
\hline & $\begin{array}{l}\text { COR2. Mezclar correctamente los objetos virtuales con el } \\
\text { mundo real. }\end{array}$ & $\checkmark$ & $\checkmark$ & $\checkmark$ & $\checkmark$ & 4 \\
\hline & COR3. Adaptar los objetos de RA al entorno del usuario. & $\checkmark$ & $\checkmark$ & $\checkmark$ & $\checkmark$ & 4 \\
\hline & COR4. Mostrar realismo en los objetos de RA. & $\checkmark$ & $\checkmark$ & $\checkmark$ & $\checkmark$ & 4 \\
\hline & COR5. Ofrecer superposición precisa de RA basada en GPS. & $\checkmark$ & $\checkmark$ & $\checkmark$ & & 3 \\
\hline & $\begin{array}{l}\begin{array}{l}\text { COR6. Mostrar la superposición } \\
\text { correctamente. }\end{array} \\
\text { col modelo }\end{array}$ & $\checkmark$ & $\checkmark$ & $\checkmark$ & $\checkmark$ & 4 \\
\hline
\end{tabular}




\section{CONCLUSIONES Y TRABAJO FUTURO}

El diseño de UX es un aspecto que adquiere cada vez más importancia en las aplicaciones de Turismo Cultural con RA. Teniendo en cuenta que las pautas de usabilidad universal ofrecen enfoques muy genéricos y no cubren todos los aspectos de un dominio, resulta necesario crear recomendaciones de diseño específicas para este dominio.

La principal contribución de este trabajo es el conjunto de guías de diseño UX para aplicaciones móviles de Patrimonio Cultural con Realidad Aumentada. Sin embargo, el conjunto de guías de diseño obtenido tiene algunas limitaciones; si bien las guías han sido sometidas al análisis de 4 expertos, aún no se han validado aplicándolas en el diseño de aplicaciones concretas.

Como trabajo futuro, se proyecta ampliar el proceso de revisión, incluyendo otros trabajos. También se propone refinar y validar este conjunto de guías por medio de su aplicación en casos de estudio. Futuras propuestas incluyen completar la especificación de cada una de las guías.

\section{AGRADECIMIENTOS}

A la UNPA por el soporte al Proyecto de Investigación 29/B256, denominado: "Contextos Digitales para Asistencia de los Ciudadanos: Enfoques de Experiencia de Usuario". A las expertas en UX del Grupo de Investigación y Formación en Ingeniería de Software (GIFIS).

\section{REFERENCIAS}

ATTILA, K., \& EDIT, B. (2012). Beyond reality: The possibilities of augmented reality in cultural and heritage tourism. 2nd International Tourism and Sport Management Conference, 5 (6).

AZUMA, R. T. (1997). A survey of augmented reality. Presence: Teleoperators \& Virtual Environments , 6 (4), 355-385. https://doi.org/10.1162/pres.1997.6.4.355

BEKELE, M. K. PIERDICCA, R., FRONTONI, E., MALINVERNI, E. S., \& GAIN, J. (2018). A survey of augmented, virtual, and mixed reality for cultural heritage. Journal on Computing and Cultural Heritage (JOCCH) , 11 (2), 1-36. https://doi.org/10.1145/3145534

BOBOC, R. G., DUGUleANĂ, M., VOINEA, G. D., POSTELNiCU, C. C., POPOVICI, D. M., \& CARROZZINO, M. (2019). Mobile augmented reality for cultural heritage: Following the footsteps of Ovid among different locations in Europe. Sustainability, 11 (4), 1167. https://doi.org/10.3390/su11041167

CARDOZO, C., MARTIN, A., SALDAÑO, V., \& GAETÁN, G. (2020). Análisis de herramientas de Realidad Aumentada para crear experiencias de turismo. VI Encuentro de Investigadores, Becarios y Tesistas de la Patagonia Austral, (págs. 710-714).

CARMIGNIANI, J., FURHT, B., ANISETTI, M., CERAVOLO, P., DAMIANI, E., \& IVKOVIC, M. (2011). Augmented reality technologies, systems and applications. Multimedia tools and applications , 5 (1), 341-377. https://doi.org/10.1007/s11042010-0660-6 
CONDE, S. B. (2020). Nuevas tecnologías y difusión del turismo cultural: descubriendo a Goya con realidad aumentada. Rotur: revista de ocio y turismo , 14 (1), 81-93. https://doi.org/10.17979/rotur.2020.14.1.5945

DAHLAN, R. A. (2016). Software Requirements for Mobile Augmented Reality Tourism Application. Journal of Information Systems Research and Innovation, 10 (3), 9-14.

DEY, A., BILLINGHURST, M., LINDEMAN, R. W., \& SWAN, J. (2018). A systematic review of 10 years of augmented reality usability studies: 2005 to 2014. Frontiers in Robotics and AI (5), 37. https://doi.org/10.3389/frobt.2018.00037

EL-HAKIM, S. F., BERALDIN, J. A., PICARD, M., \& GODIN, G. (2004). Detailed 3D reconstruction of large-scale heritage sites with integrated techniques. IEEE computer graphics and applications , 24 (3), 21-29. https://doi.org/10.1109/MCG.2004.1318815

FIERRO, F. A., MANOSALVAS, C. A., \& HIDROBO, S. (2018). Comparativa técnica de herramientas para realidad aumentada: wikitude, vuforia y artoolkit . Revista Científica Axioma (19), 86-96. https://doi.org/10.26621/XV19.2018.12.A08. PUCESI.2550.6684

FRITZ, F., SUSPERREGUI, A., \& LINAZA, M. T. (2005). Enhancing cultural tourism experiences with augmented reality technologies. 6th International Symposium on Virtual Reality, Archaeology and Cultural Heritage (VAST).

HAN, D. I., \& JUNG, T. (2018). Identifying tourist requirements for mobile AR tourism applications in urban heritage tourism. En Augmented Reality and Virtual Reality (págs. 3-20). Springer, Cham. https://doi.org/10.1007/978-3-319-64027-3_1

HAN, D. I., TOM DIECK, M. C., \& JUNG, T. (2018). User experience model for augmented reality applications in urban heritage tourism. Journal of Heritage Tourism , 13 (1), 46-61. https://doi.org/10.1080/1743873X.2016.1251931

HERPICH, F., GUARESE, R. L., \& TAROUCO, L. M. (2017). A comparative analysis of augmented reality frameworks aimed at the development of educational applications. Creative Education , 8 (09), 1433. https://doi.org/10.4236/ce.2017.89101

ISO/IEC JTC 1/SC 7. (2011). ISO/IEC 25010:2011. Software engineering - Software product Quality Requirements and Evaluation (SQuaRE) - System and software quality models. International Organization for Standardization.

ISO/TC 159/SC 4. (2010). ISO 9241-210:2010. Ergonomics of Human-System InteractionPart 210: Human-Centred Design for Interactive Systems.

JOOSTE, D., RAUTENBACH, V., \& COETZEE, S. (2015). Results Of and Evaluation Of Augmented Reality Mobile Development Frameworks for Addresses in Augmented Reality. Free and Open Source Software for Geospatial (FOSS4G) Conference Proceedings, 15.

KENNETH, R. O. (1997). Web style guides: who, what, where. 15th annual international conference on Computer documentation (SIGDOC '97) (págs. 189-197). New York, NY, USA: Association for Computing Machinery.

LIM, K. C., SELAMAT, A., ALIAS, R. A., KREJCAR, O., \& FUJITA, H. (2019). Usability Measures in Mobile-Based Augmented Reality Learning Applications: A Systematic Review. Applied Sciences , 9 (13), 2718. https://doi.org/10.3390/app9132718

LUNA, U., ETXEBERRIA, A. I., \& GRACIA, M. P. (2019). El patrimonio aumentado. 8 apps de Realidad Aumentada para la enseñanza-aprendizaje del patrimonio. Revista interuniversitaria de formación del profesorado (94), 43-62.

MASIP, L., MARTINIE, C., WINCKLER, M., PALANQUE, P., GRANOLLERS, T., \& OLIVA, M. (2012). A design process for exhibiting design choices and trade-offs in (potentially) conflicting user interface guidelines. International Conference on Human-Centred Software Engineering (págs. 53-71). Berlin: Springer. https://doi.org/10.1007/978-3-642-34347-6_4 
MILGRAM, P., \& KISHINO, F. (1994). A taxonomy of mixed reality visual displays. IEICE Transactions on Information and Systems , 77 (12), 1321-1329.

NARCISO, D., PÁDUA, L., ADÃO, T., PERES, E., \& MAGALHÃES, L. (2015). MixAR mobile prototype: visualizing virtually reconstructed ancient structures in situ. Procedia Computer Science (64), 852-861. https://doi.org/10.1016/ j.procs.2015.08.638

NIELSEN, J. (1994). Usability inspection methods. Conference companion on Human factors in computing systems, (págs. 413-414). https://doi.org/10.1145/259963.260531

PORTALÉS, C., LERMA, J. L., \& NAVARRO, S. (2010). Augmented reality and photogrammetry: A synergy to visualize physical and virtual city environments. ISPRS Journal of Photogrammetry and Remote Sensing , 65 (1), 134-142. https://doi.org/10.1016/j.isprsjprs.2009.10.001

QUIÑONES, D., RUSU, C., \& RUSU, V. (2018). A methodology to develop usability/user experience heuristics. Computer Standards \& Interfaces (59), 109-129. https://doi.org/10.1016/j.csi.2018.03.002

SYLAIOU, S., MANIA, K., LIAROKAPIS, F., WHITE, M., WALCZAK, K., WOJCIECHOWSKI, R., Y OTROS. (2015). Evaluation of a cultural heritage augmented reality game. Cartographies of Mind, Soul and Knowledge .

TORRES, D. R. (2013). La realidad aumentada y su aplicación en el patrimonio cultural. Ediciones Trea.

VEGA, M., GAETAN, G., MARTIN, A., \& SALDAÑO, V. (2020). Realidad Aumentada para mejorar experiencias de Turismo Cultural. VI Encuentro de Investigadores, Becarios y Tesistas de la Patagonia Austral, (págs. 787-791).

VLAHAKIS, V., IOANNIDIS, M., KARIGIANNIS, J., TSOTROS, M., GOUNARIS, M., STRICKER, D., Y OTROS. (2002). Archeoguide: an augmented reality guide for archaeological sites. IEEE Computer Graphics and Applications , 22 (5), 52-60. https://doi.org/10.1109/MCG.2002.1028726

VLAHAKIS, V., PLIAKAS, T., DEMIRIS, A. M., \& IOANNIDIS, N. (2003). Design and Application of an Augmented Reality System for continuous, context-sensitive guided tours of indoor and outdoor cultural sites and museums. 4th International Symposium on Virtual Reality, Archaeology and Intelligent Cultural Heritage (VAST ), (págs. 155164).

ZHAO, Q. (2009). A survey on virtual reality. Science in China Series F: Information Sciences , 52 (3), 348-400. https://doi.org/10.1007/s11432-009-0066-0 